\title{
Summary: Gravitation and Cosmology
}

\author{
Maurício O. Calvão \\ Instituto de Física, Universidade Federal do Rio de Janeiro, \\ Caixa Postal 68528, CEP 21941972 Rio de Janeiro, RJ, Brazil
}

Received on 6 August, 2006

\begin{abstract}
The XXVI Encontro Nacional de Física de Partículas e Campos (ENFPC: Brazilian National Meeting on Particles and Fields) took place in São Lourenço, MG, Brazil, in October 2005. I was invited to deliver the summary talk on the area of gravitation and cosmology, in which I took the opportunity to briefly present a historic sketch of the ideas related to relativity, gravitation and cosmology in general, then some admittedly idiosyncratic highlights on current research in the field and an overview of some aspects of the corresponding national situation.
\end{abstract}

Keywords: Gravitation; Cosmoloy; Relativity

\section{GRAVITATION AND COSMOLOGY IN GENERAL}

\section{A. Historical perspective}

As usual, we can find some emergent relevant ideas related to the areas of relativity, gravitation and cosmology at most in ancient Greece. If one is in the mood of digging deeper, the magical or mythological world views of other ancient civilizations, such as, e.g., Sumerian, Chinese, Norse, or Mayan, are worth being recollected and an extremely enjoyable narrative on this and other topics of mainly modern cosmology for the learned reader is provided in the excellent thought-provoking book by E. R. Harrison [1]. Here we just want to call attention to the theoretical or philosophical system of Aristotle of Stagirus ( 384 BC $-\sim 322$ BC), which somehow was the culmination of Greek science. At least three ideas are relevant for us in the Aristotelian system: (i) geocentrism, (ii) the division of physics, or rather mechanics, into terrestrial and celestial, and (iii) circles as natural or privileged trajectories or motions. These were all doomed to be superseded by later physics, particularly relativity and modern cosmology.

The deconstruction of Aristotelian physics is conventionally agreed to have started with N. Copernicus (1473 - 1543), whose main work, Revolutions of the celestial spheres, revived the heliocentric ideas of Aristarchus of Samos ( 310 $\mathrm{BC}-\sim 230 \mathrm{BC}$ ) and had a tremendous impact. He dethroned Earth from the center of the universe, but still maintained the circles as (celestial) privileged trajectories. Shortly thereafter, G. Galilei (1564 - 1642) presented, on his Dialogue concerning the two chief world systems, what came to be known as the Galilean principle of relativity (as applied to mechanical phenomena). There followed J. Kepler (1571 - 1630), who, by inheriting the meticulous observations of T. Brahe (1546 1601), was able to, after a painstaking work of around twenty years, establish his three famous laws of planetary motion.

Then, I. Newton (1643 - 1727), possibly the greatest scientist of all times, invented the basis of modern mathematics, the infinitesimal calculus, as well as much of modern physics, with his mechanics, gravitation and optics. He stated the three famous laws of the now called classical (Newtonian) mechanics, thereby incorporating the Galilean principle of relativity. He was able to derive Kepler's laws from his mechanics, and so unified terrestrial and celestial mechanical motions, and erected the uniform straight motion as the natural one.

And thus, very schematically, was paved the way to the socalled theories of relativity and to modern relativistic cosmology, all of them essentially formulated by A. Einstein (1879 $-1955)$.

\section{B. Relativity in a nutshell}

In essence, the special theory of relativity is physics in the flat background of Minkowski space-time. Due to its isometries, there is associated with every frame of reference (which is a congruence of time-like curves or a unit time-like vector field) a family of other frames which are physically equivalent to the original one; this means that every conceivable experiment which can be performed and analyzed from the point of view of any of these frames will, given the same corresponding initial or boundary conditions, lead to the same qualitative and quantitative results. The particularly simple and privileged inertial frames of reference are those whose kinematical parameters vanish identically: they are constituted by a congruence of world-lines, which are geodesic (excluding Rindler's frame, e.g.), have no expansion (excluding Milne's frame, e.g.), no vorticity (excluding "rotating disk", e.g.) and no shear.

On the other hand, the so-called general theory of relativity is physics in a non-flat space-time (curvature being the legitimate sign of real gravitational fields), whose metric obeys Einstein's field equations. In my opinion, as cogently defended by V. Fock (1898 - 1974) in [2], the phrase "general relativity" is somewhat misleading, because, interpreted as in the above paragraph, there can be no more general principle of relativity than the special one, since this is equivalent to the existence of an isometry group with more than 10 parameters and this is impossible in a four-dimensional space-time. Perhaps general relativity should be rechristened as relativistic theory of gravitation...

Einstein's main achievement in his so-called special and general theories of relativity was the physicalization of geometry. He changed the causal structure of space-time (the Newtonian three-dimensional present was turned into a relativistic 
four-dimensional present or elsewhere, due to the existence of an invariant bounded speed of signal propagation), the natural motions (from the Newtonian inertia principle to relativistic geodesics) and dynamicized space-time through the field equations

$$
G^{\alpha \beta}=8 \pi G T^{\alpha \beta}
$$

\section{Experimental/observational status highlights}

\section{Special relativity}

A beautiful kinematic test theory of special relativity was devised by Mansouri \& Sexl, in 1976 [3]. If, in a given "preferred" frame $S$ (the CMB one, e.g.), the velocity of light is $c=1$ (in appropriate units), then the generalized Lorentz transformations are described by

$$
\begin{aligned}
t^{\prime} & =a(v) t+\vec{\varepsilon}(\vec{v}) \cdot \vec{x}^{\prime} \\
\vec{x}^{\prime} & =d(v) \vec{x}+[b(v)-d(v)](\hat{n} \cdot \vec{x}) \hat{n}+b(v) \vec{v} t
\end{aligned}
$$

where the three-vector $\vec{v}$ is the relative velocity of frame $S^{\prime}$ with respect to frame $S$ and $\hat{n}:=\vec{v} / v$. Specifically, it is easy to check that $a(v)$ governs the time dilation, $b(v)$ the longitudinal length contraction, $d(v)$ the transverse length contraction, and $\vec{\varepsilon}(\vec{v})$ the clock sinchronization; in the usual Einsteinsynchronized special relativity framework, these functions are, of course: $a_{S R}(v)=1 / \gamma(v)=\left(1-v^{2}\right)^{1 / 2}, b_{S R}(v)=\gamma(v)$, $d_{S R}(v)=1, \vec{\varepsilon}_{S R}(\vec{v})=-\vec{v}$. From these expressions, we may calculate the speed of light in the new frame; under Einsteinsynchronization of the clocks, it turns out to be anisotropic and dependent on the relative speed of the frames as well, according to:

$$
\begin{aligned}
c^{\prime}(\theta, v)= & 1+(\beta-\delta-1 / 2) v^{2} \sin ^{2} \theta \\
& +(\alpha-\beta+1) v^{2}+O\left(v^{4}\right) .
\end{aligned}
$$

Here we have expanded the (constant) parameters $\alpha, \beta, \delta$ from the expansion of $a(v), b(v), d(v)$ up to second order in the relative speed $v$, that is:

$$
\begin{aligned}
& a(v)=1+\alpha v^{2}+O\left(v^{3}\right), \\
& b(v)=1+\beta v^{2}+O\left(v^{3}\right), \\
& d(v)=1+\delta v^{2}+O\left(v^{3}\right) .
\end{aligned}
$$

It should be borne in mind that, for the usual special relativity case,

$$
\begin{aligned}
& \alpha_{S R}=-1 / 2, \\
& \beta_{S R}=1 / 2, \\
& \delta_{S R}=0 .
\end{aligned}
$$

Modern experiments try to detect any deviations from these values, mainly through the use of optical or microwave resonators (for the case of our speed relative to the CMB frame, we have $v \simeq 370 \mathrm{~km} / \mathrm{s} \simeq 1.23 \times 10^{-3} c$ ). The best fits (and $1 \sigma$ confidence levels) or upper limit are [4]:

$$
\begin{aligned}
-\beta+\delta+1 / 2 & =(2.2 \pm 1.5) \times 10^{-9}, \\
-\alpha+\beta-1 & =(1.6 \pm 3.0) \times 10^{-7}, \\
\alpha+1 / 2 & <2.2 \times 10^{-7} .
\end{aligned}
$$

\section{General relativity}

The so-called classical tests of general relativity (gravitational spectral shift or Doppler effect, gravitational deflection of electromagnetic waves, and perihelion precession) are relatively imprecise tests; however, the experimentalists have been creative enough in drawing the border a little further. I would just like to mention the experiment with a rocket-borne hydrogen maser clock [5] to check the generic Doppler shift formula,

$$
\frac{v_{D}}{v_{E}}=\frac{\left.k^{\alpha} u_{\alpha}\right|_{D}}{\left.k^{\beta} u_{\beta}\right|_{E}},
$$

which confirmed the theoretical result to a precision of $10^{-4}$, and the very long baseline radiointerferometric experiment [6] to check the gravitational deflection formula,

$$
\delta \phi \simeq \frac{4 G M}{c^{2} b},
$$

which confirmed the theoretical result also to a precision of $10^{-4}$.

Gravitational waves The really most active area in experimental/observational pure gravitation research is undoubtedly gravitational wave or radiation detection (LIGO, LISA) [7]. There are several worldwide experiments under operation, construction or design. Brazil has its share on this market, via the Schenberg detector, a spherical antenna, which, in its first commissioning run, will operate with three transducers, cooled to around $4 \mathrm{~K}$ and a target sensitivity of $h \sim 2 \times 10^{-21}$ $\mathrm{Hz}^{-1 / 2}$ in a $60 \mathrm{~Hz}$ bandwidth around $3.2 \mathrm{kHz}$ [8]. Possibly the main advantage of this kind of spherical antenna is its ability to detect a gravitational wave from any direction and with arbitrary polarization. The principal source candidates for such waves seem to be, primarily, neutron star instabilities and inspiralling of mini-black hole binaries, or, to a lesser extent, e.g., core collapse in supernova events and coalescence of neutron stars and/or black holes [9].

\section{Cosmology}

Recently, I guess it is fair to say that the scene in physics has been frequently stolen by cosmology; three sets of observations, at least, account for that, all of them heralding an age of data abundance (if not precision) cosmology: (i) supernovae Ia surveys, which apparently revealed an unexpected acceleration of the cosmic expansion, (ii) wide-field surveys of galaxies and quasars, which revealed an a complex spatial 
distribution of matter in filaments, voids, and walls, and (iii) the cosmic microwave background radiation, which is one of the cleanest and deepest probes of cosmic history, since primordial epochs some ten billion years ago. There is a new paradigmatic standard cosmological model: the flat adiabatic cold dark matter model, with around $70 \%$ of dark energy, $25 \%$ of cold dark matter, and only $5 \%$ baryonic matter; it is able to consistently explain all different observational data (primordial cosmonucleosynthesis, matter perturbations, etc) [10].

Cosmic microwave background radiation Again, it is quite easy to identify the cosmic microwave background radiation field as one of the hottest in experimental/observational cosmology (WMAP, Planck); current outstanding related research topics are polarization, integrated Sachs-Wolfe and Sunyaev-Zeldovich effects [11]. Here, as with gravitational waves, there are several ongoing or planned experiments, and there is an active Brazilian group, with a long tradition in the field; their most recent collaboration was the Background Emission Anisotropy Telescope (BEAST), which provided several $\mathrm{CMB}$ maps, from which, e.g., angular power spectra and foreground contamination results were established $[12,13]$.

\section{GRAVITATION AND COSMOLOGY IN BRAZIL AND IN THE XXVI ENFPC}

\section{A. Geographical distribution in the country}

To get a gross feeling for the geographical distribution of the reseachers in gravitation and cosmology across the nation, I performed a search within the 2004 census database of the directory of research groups of the Lattes platform from $\mathrm{CNPq}$, the major federal sponsoring agency of Brazil. I looked for the words "relatividade" (relativity) or "gravitação" (gravitation) or "cosmologia" (cosmology) in the fields "Nome do grupo" (Group name) or "Linha de pesquisa" (Research line) in the great knowledge area of "Ciências Exatas e da Terra" (Exact and Earth Sciences). The grand total found was thus 47 groups, divided by geographical regions as: (a) Southeast: 26; (b) Northeast: 9; (c) Centerwest: 5; (d) South: 4, and (e) North: 3 .

\section{B. Executive summary of the meeting}

From a total of 323 officially registered participants, 75 (23\%) presented some work related to the area of gravitation and cosmology.

Of the six plenary talks, there were two $(1 / 3)$ dedicated to the area: the first one, entitled "The present status of quantum field theory in curved spacetime", by R. Wald (University of Chicago, USA). There he called attention once again to the untenability ot a consistent notion of particle in a curved spacetime, and made a call of arms to import in earnest the techniques of microlocal analysis in order to formulate a reasonably coherent framework of interacting fields in curved backgrounds. The second plenary talk, entitled "Polarization in the cosmic microwave background", was delivered by $\mathrm{J}$. Bartlett (APC - Université Paris 7, France). There he reviewed both the theory and the experiments related to this extremely timely topic; it holds the promise to vindicate the inflationary paradigm, if the so-called $B$ or curl modes of polarization in $\mathrm{CMB}$ are really detected, since they are the smoking gun of inflation-induced gravitational waves.

As concerns the twelve parallel talks, three (1/4) of them dealt with themes on gravitation and cosmology: "Cosmology: from dark matter and energy to extra dimensions", by J. Alcaniz (Observatório Nacional, Brazil), "Brane cosmology”, by E. Abdalla (Universidade de São Paulo, Brazil), and "Semi-classical-gravity effects driving the late acceleration of the Universe", by D. Vanzella (Universidade de São Paulo, Brazil).

There was a scientific outreach lecture, by M. Gleiser (Dartmouth College), entitled "O que sabemos e o que não sabemos sobre o Universo" ("What we do and do not know about the Universe"), which attracted an enormous attendance of people from the town.

Last, but by far not least, among 73 oral communications, there were $18(\sim 25 \%)$ on gravitation and cosmology, and, among 259 poster or panels, $54(\sim 21 \%)$ were on gravitation and cosmology. Table I shows how many of those may classified as theoretical or experimental works.

\begin{tabular}{|c|c|c|}
\hline & $\begin{array}{c}\text { oral } \\
\text { communications }\end{array}$ & $\begin{array}{c}\text { posters } \\
\text { (panels) }\end{array}$ \\
\hline gravitation: theory & 8 & 25 \\
gravitation: experiment & 1 & 4 \\
cosmology: theory & 7 & 19 \\
cosmology: experiment & 2 & 6 \\
\hline
\end{tabular}

TABLE I: Division of presentations into theoretical or experimental.

\section{CONCLUSION}

For concluding remarks, I would like to stress three main points:

1. claim for a greater share of the national scientific budget in the area of gravitation and cosmology, mainly directed to observational or experimental activities. In this regard, the initiatives related to the gravitational wave and cosmic microwave background should be reinforced and the new Dark Energy Survey should be decisively supported.

2. there is too high a concentration of groups in the southeast region of Brazil, mainly in fact in RJ and SP. The spreading of young scientists across other regions of the country should be firmly sponsored.

3. there is still too high a bias towards purely theoretical aspects, without taking advantage of the abundant 
existent observations and experimental results, specifically in cosmology. The interaction with the astrophysical community and the exploitation of available public databases should be strongly promoted.
[1] E. R. Harrison, Cosmology, the science of the universe, 2nd. ed. Cambridge University Press, Cambridge, England, 2000.

[2] V. A. Fock, The theory of space, time and gravitation, 2nd. rev. ed. Pergamon Press, Oxford, England, 1966.

[3] R. Mansouri and Sexl, "A test theory of special relativity. I: simultaneity and clock synchronization", Gen. Relat. Gravit. 8, 497 (1977); see also D. Giulini and N. Straumann, "Einstein's impact on the physics of the twentieth century", physics/0507107.

[4] P. Wolf et alii, "Recent experimental tests of special relativity", physics/0506168.

[5] R. F. C. Vessot et alii, "Test of relativistic gravitation with a space-born hydrogen maser", Phys. Rev. Lett. 45, 2081 (1980).

[6] D. S. Robertson, W. E. Carter, and W. H. Dillinger, "New measurement of solar gravitational deflection of radio signals using VLBI", Nature 349, 768 (1991).

[7] K. S. Thorne, "Gravitational waves: a web-based course", http://elmer.tapir.caltech.edu/ph237/.
[8] O. D. Aguiar et alii, "The Brazilian gravitational wave detector Mario Schenberg: a status report", Class. Quantum Grav. 23, S239 (2006).

[9] J. C. N. de Araújo, O. D. Miranda, and O. D. Aguiar, "Detectability of $f$-mode unstable neutron stars by the Schenberg spherical antenna", Class. Quantum Grav. 22, S471 (2005).

[10] To be honest, the jury is still out as to whether the real cause of the present acceleration of the universe is dark energy or alternative gravitational theories.

[11] S. Dodelson, Modern cosmology. Academic Press, San Diego, USA (2003).

[12] P. R. Meinhold et alii, "A map of the cosmic microwave background from the BEAST experiment", Astrophys. J. Suppl. 158, $101(2005)$.

[13] I. J. O'Dwyer et alii, "The cosmic microwave background anisotropy power spectrum from the BEAST experiment", Astrophys. J. Suppl. 158, 93 (2005). 